\title{
A clinical investigation into long term efficacy outcomes in the management of severe canine osteoarthritis (OA) using an optimised regenerative medicine (RM) approach.
}

\section{Joanna Miller ${ }^{1}$, Christine Standen $^{1}$, Alex Georgiou ${ }^{2}$, Andrew Armitage ${ }^{3}$}

${ }^{1}$ Cell Therapy Sciences, Coventry, United Kingdom. ${ }^{2}$ Coventry University, Coventry, United Kingdom. ${ }^{3}$ Greenside Regenerative Therapies, Greenside Vets, St Boswells, United Kingdom

Objectives: To evaluate whether optimized regenerative medicine protocols can provide long-term control of severe OA in dogs.

\begin{abstract}
Methods: Dogs with a well-documented history of moderate and severe $O A$ were given intra-articular (IA) injections of culture expanded adipose derived autologous stem cells (adMSCs; AdiShot ${ }^{\mathrm{TM}}$ ) either alone or with a centrifuge prepared pure platelet rich plasma (PRP) and/or laser therapy as part of an individualized RM treatment plan. A Veterinary Global Score (VGS) based on clinical examinations vy a veterinarian and owners' feedback on pain and mobility (CBPI and/or LOAD scores), was used to evaluate treatment response during a follow-up period of up to 4 years. Long-term outcomes in severe disease were evaluated in a sub-group of dogs. The individualized RM protocols were repeated at the discretion of the vet, when Global Scores indicated a return of pre-treatment symptoms.
\end{abstract}

Figure 2: Typical response pattern from 2 representative cases studies showing VGS, CBPI and LOAD scores before treatment and at various times up to 15 months after RM treatment.

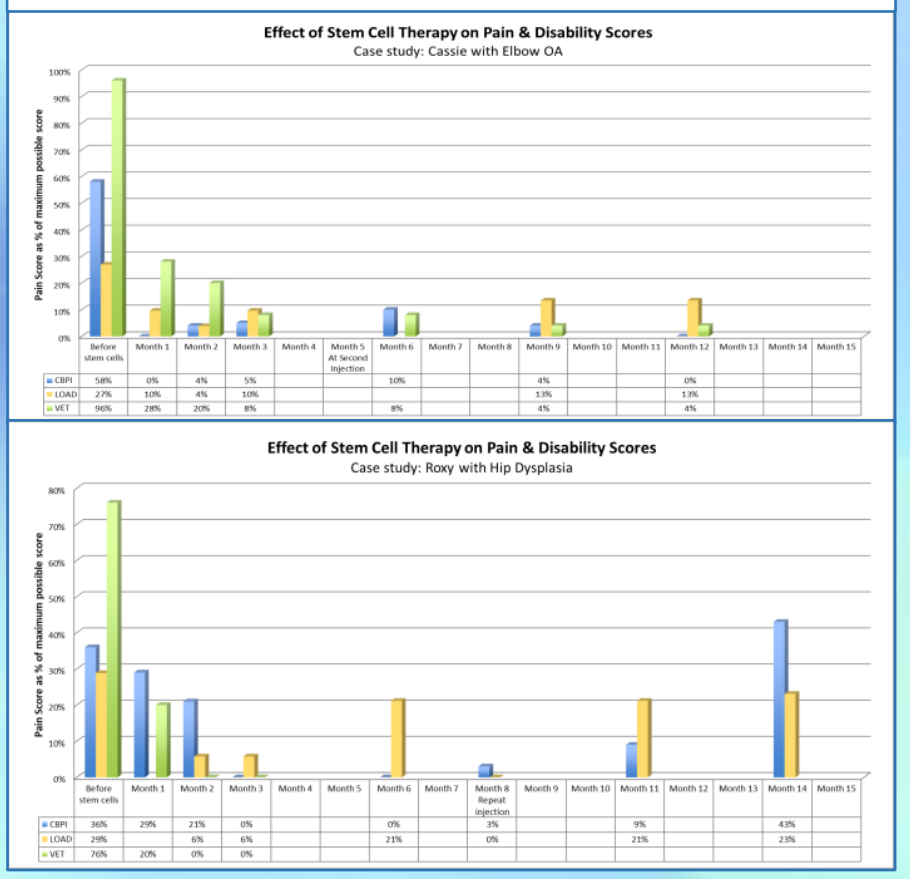

Figure 1: Distribution of dogs with severe OA responding to RM treatment (assessed by VGS)

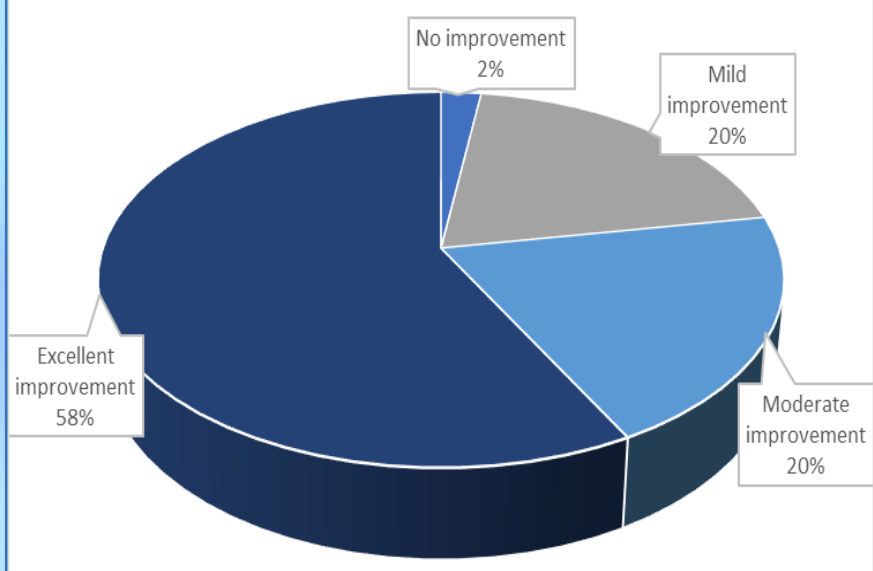

Results: 499 dogs were entered into the main analysis. Overall clinical response was $79 \%$ moderate-excellent response, measured by the VGS. During the follow-up period a total of $29 \%$ of all dogs required repeat stem cells/RM protocol and the mean time to repeat treatment was 11 months.

45 dogs with severe OA provided long-term follow-up data for inclusion into sub-group analysis. The majority of dogs (98\%) with severe OA demonstrated a clinical improvement (44/45 cases) following the RM treatment protocol, with $58 \%$ pf this severe OA sub-group evaluated as demonstrating an excellent response (26/45).

Of these 45 dogs with severe OA, long term follow-up showed that at total of $58 \%(26 / 45)$ required repeat treatment with a mean time to repeat therapy of 15.6 months.

Of the dogs that received a repeat treatment, 15 out of 26 went on to receive a second repeat treatment. The average time between repeat treatments was 12.4 months.

Statement (conclusions): Following administration of culture-expanded stem cells as part of an individualised RM protocol, $98 \%$ of dogs with severe OA demonstrated a clinical improvement that was maintained and well tolerated for between 1 and 4 years using a long-term management protocol. Further studies are required to evaluate this effect size in comparison with other long term OA management protocols. 